\title{
Separating exchange splitting from spin mixing in gadolinium by femtosecond laser excitation
}

\author{
Beatrice Andres, ${ }^{*}$ Marc Christ, Cornelius Gahl, Marko Wietstruk, and Martin Weinelt \\ Freie Universität Berlin, Fachbereich Physik, Arnimallee 14, 14195 Berlin, Germany \\ Jürgen Kirschner \\ Max-Planck-Institut für Mikrostrukturphysik, Weinberg 2, 06120 Halle/Saale, Germany
}

(Dated: October 16, 2015)

\begin{abstract}
Employing spin-, time-, and energy-resolved photoemission spectroscopy, we present the first study on the spin polarization of a single electronic state after ultrafast optical excitation. Our investigation concentrates on the majority-spin component of the $d$-band-derived $\operatorname{Gd}(0001)$ surface state $d_{z^{2}}^{\uparrow}$. While its binding energy shows a rapid Stoner-like shift by $90 \mathrm{meV}$ with an exponential time constant of $\tau_{\mathrm{E}}=0.6 \pm 0.1 \mathrm{ps}$, the $d_{z^{2}}^{\uparrow}$ spin polarization remains nearly constant within the first picoseconds and decays with $\tau_{\mathrm{S}}=15 \pm 8 \mathrm{ps}$. This behavior is in clear contrast to the equilibrium phase transition, where the spin polarization vanishes at the Curie temperature.
\end{abstract}

PACS numbers: 78.47.-p, 71.45.Gm, 73.20.At

Laser excitation is capable of creating new transient states of matter, that are not reached on equilibrium pathways [1-6]. In magnetically ordered materials this opens new routes to the ultrafast manipulation of the magnetic order [7-10]. Investigating the underlying processes provides a yet unseen view on the very basics of magnetism such as the relation between the electronic and magnetic structure. Contrary to thermal heating, laser excitation drives the electronic, phonon, and spin subsystems out of equilibrium [11]. It may therefore lead to a response quite different from the equilibrium magnetic phase transition as we show in this letter.

The first experiment on the laser-induced demagnetization of Gd metal applied spin-resolved photoemission on the nanosecond timescale [12]. A decrease of the spin polarization of the secondary electrons was observed, which was explained by spin-lattice relaxation with a characteristic time of $100 \pm 80 \mathrm{ps}$. This result was corroborated by measurements of the magnetic linear dichroism (MLD) observed in $4 f$ core-level photoemission [13]. Later studies with sub-picosecond time resolution revealed an additional much faster decrease of magnetic figures: The X-ray magnetic circular dichroism (XMCD) at the $M_{5}$ edge decreases within $0.76 \pm 0.25 \mathrm{ps}$ [14]. So do rotation and ellipticity of the magneto-optical Kerr effect (MOKE) $[15,16]$ as well as the $(5 d 6 s)$ valence-band exchange-splitting $[17,18]$. A binding energy shift on this timescale has also been found for the majority-spin component of the surface state $[19,20]$. In contrast, the magnetic component of the surface-sensitive second harmonic generation ( $\mathrm{SHG}$ ) shows an immediate response within the laser pulse duration of $100 \mathrm{fs}[19,21,22]$. Whereas a recent photoemission experiment reveals again a ps response of the MLD in the $4 f$ states [23]. Obviously different observables of the magnetization dynamics suggest significantly different response times, while in the equilibrium phase transition all techniques map the same behavior. Furthermore, most of the femtosecond pump-probe experiments are not consistent with the spin-resolved measurements of Ref. 12 , which may be attributed to its 10-ns pump-pulse duration. It is thus still unclear on which timescale we can modify the spin polarization in Gd using an ultrafast laser pulse.

In this letter we present a comparative study of equilibrium vs. laser-induced demagnetization of Gd using spin-resolved photoemission. Upon excitation with a $48-$ fs laser pulse the majority-spin component $d_{z^{2}}^{\uparrow}$ of the Gd surface state shifts towards the Fermi level, reducing the exchange splitting on a sub-ps timescale. In contrast, the $d_{z^{2}}^{\uparrow}$ spin polarization changes only slightly with a one order of magnitude slower ps dynamics.

For pump we use $s$-polarized pulses at the fundamental photon energy of $1.6 \mathrm{eV}$ of a $300-\mathrm{kHz}$ Ti:Sapphire regenerative amplifier (RegA, Coherent) incident at an angle of $45^{\circ}$ off normal along the $\mathrm{Gd}[1000]$ direction. The absorbed pump fluence was $3.9 \mathrm{~mJ} / \mathrm{cm}^{2}$. A time-delayed $p$ polarized ultraviolet probe is generated by frequency quadrupling the fundamental to $6.3 \mathrm{eV}$. The temporal resolution is $\leq 70 \mathrm{fs}$ as determined from fitting the rising edge of the electronic temperature (Fig. 3 (a)). A cylindric sector analyzer (CSA 300, Focus) transmits the photoemitted electrons to the spin detector with an energy and angular resolution of $65 \mathrm{meV}$ and $\pm 2.5^{\circ}$, respectively. We use an exchange-scattering-based spin detector [24], which supports two measurement modes: A spin-integrated mode, where all incoming electrons impinge directly on a channeltron, and a spin-resolved mode, where we place an oxygen-passivated $\mathrm{Fe} / \mathrm{W}(001)$ target into the electron beam for spin-dependent exchange scattering at $6 \mathrm{eV}$ kinetic energy into a second channeltron in back-reflection geometry. The spin detector has a figure of merit of $2.2 \cdot 10^{-3}$ with an intensity loss of 2 orders of magnitude [24]. We took advantage of switching on the fly between spin-integrated and spin-resolved mode, to ac- 


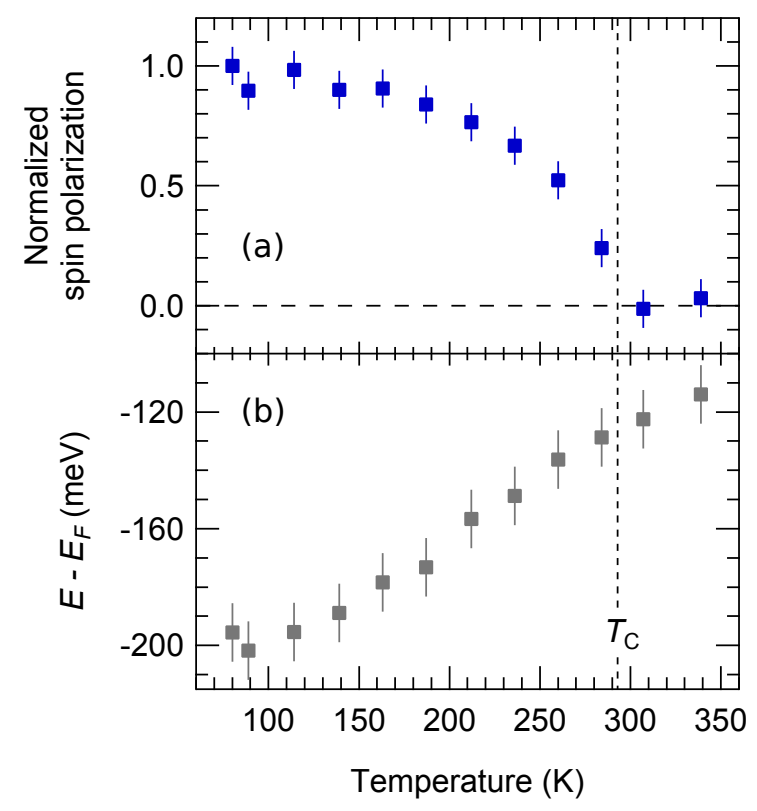

FIG. 1. Spin polarization (a) and binding energy (b) of the majority-spin surface state $d_{z^{2}}^{\uparrow}$ in the ferro- to paramagnetic phase transition in thermal equilibrium. The spin polarization vanishes at $T_{\mathrm{C}}=293 \mathrm{~K}$ but the exchange splitting remains finite.

quire high-intensity spin-integrated data on the energetic behavior and valuable information on the spin polarization in the same experiment. The investigated Gd(0001) samples were prepared by evaporating $100 \AA$ of Gd onto a W(110) substrate at a temperature of $300 \mathrm{~K}$, a deposition rate of $5 \AA / \mathrm{min}$ and a pressure of $6 \times 10^{-10} \mathrm{mbar}$. Beforehand, the substrate was cleaned from carbon impurities as described in Ref. 25. To obtain a smooth and contaminant-free surface, the Gd films were annealed for 1 minute at $780 \mathrm{~K}$. During the measurements at a pressure of $2 \times 10^{-11}$ mbar, we kept a sample temperature of $90 \mathrm{~K}$ by liquid nitrogen cooling. All spectra were taken at $\bar{\Gamma}$ under normal emission. The Gd films were magnetized remanently in plane along the Gd[1100] direction applying a field pulse of $20 \mathrm{mT}$ via a freestanding coil.

The ferro- to paramagnetic phase transition of gadolinium at the Curie temperature $T_{\mathrm{C}}=293 \mathrm{~K}$ is a prototype system to study spin-mixing vs. Stoner behavior. The latter two limiting cases turned out to be helpful for distinguishing the temperature dependence of the electronic structure of a ferromagnet in thermal equilibrium. The Stoner model [26-29] treats delocalized electronic states and predicts a gradual decrease of the exchange splitting, which collapses at $T_{\mathrm{C}}$. By contrast, spin mixing [30-34] describes localized states with a constant magnitude but fluctuating direction of the magnetic moment. In this model the exchange splitting stays constant while the spin-polarization of the states falls steadily with increasing temperature to vanish at $T_{\mathrm{C}}$.

Evidence for the existence of a finite exchange splitting above $T_{\mathrm{C}}$ has been found in the $\operatorname{Gd}(0001)$ surface state [35-39], where the majority- (minority-)spin component $d_{z^{2}}^{\uparrow}\left(d_{z^{2}}^{\downarrow}\right)$ is situated below (above) the Fermi level $E_{\mathrm{F}}$. Fig. 1 displays the temperature-dependent spin polarization (a) and binding energy $E-E_{\mathrm{F}}$ (b) of the $d_{z^{2}}^{\uparrow}$ determined by spin-resolved laser photoemission with $4.5-\mathrm{eV}$ photons. When $\mathrm{Gd}(0001)$ is heated across $T_{\mathrm{C}}$ the $d_{z^{2}}^{\uparrow}$ spin polarization collapses while its exchange splitting is only partly lowered but remains at finite values. For more than $100 \mathrm{~K}$ above $T_{\mathrm{C}}$, the $d_{z^{2}}^{\uparrow}$ remains below $E_{\mathrm{F}}$ [38] while the minority-spin part still has a binding energy above $E_{\mathrm{F}}$ [35-37]. To conclude, in the equilibrium phase transition the surface state shows spin mixing accompanied by a partly decreasing exchange splitting, i.e. a partly Stoner-like behavior.

In the following, we investigate the laser-driven phase transition. The spin-integrated photoemission intensity near $E_{\mathrm{F}}$ is shown in Fig. 2 (a) as a function of energy $E-E_{\mathrm{F}}$ and pump-probe delay. Fig. 2 (b) compares the majority- and minority-spin intensities at selected delays. Independent of spin, there is a remarkable intensity redistribution after the excitation by the pump pulse at 0 ps. Compared to the spectra before pumping at $-0.5 \mathrm{ps}$, the $d_{z^{2}}^{\uparrow}$ surface state is significantly depopulated at $0.2 \mathrm{ps}$ and electrons are excited above $E_{\mathrm{F}}$. This redistribution is attributed to an increasing electronic temperature reflected in a broadening of the Fermi edge as well as a broadening of the surface-state line-shape. In addition the maximum of the $d_{z^{2}}^{\uparrow}$ shifts to lower binding energy (indicated by the gray horizontal bars in Fig. 2 (b)). Directly after laser excitation, at a delay of $0.2 \mathrm{ps}$, the peak position is shifted by $27 \mathrm{meV}$ towards $E_{\mathrm{F}}$. This shift increases to $90 \mathrm{meV}$ at $10 \mathrm{ps}$ delay, when the population of the $d_{z^{2}}^{\uparrow}$ already recovers, most of the laser-excited electron population above $E_{\mathrm{F}}$ is decayed, and electron and phonon subsystems are equilibrated at an elevated temperature [40]. At this temperature a part of the peak is cut by the broad Fermi edge, such that the peak position obtained from a fit to the data (indicated by the gray bar) is closer to $E_{\mathrm{F}}$ as the maximum intensity visible in the spectrum [41]. Sample cooling shifts the $d_{z^{2}}^{\uparrow}$ binding energy back to the initial value and the broadening of line shape and Fermi edge further reduces as exemplified by the spectrum at $50 \mathrm{ps}$ delay. Despite these significant changes, the ratio of majority- and minority-spin intensity and thus the spin polarization alters only weakly for all displayed pump-probe delays.

For a more detailed discussion, the temporal evolutions of the above-mentioned parameters: electronic temperature, surface-state binding energy, and spin polarization are shown in Fig. 3. The data were obtained from a fit to the spectra as described in the supplemental material [41]. The spin polarization calculates as $P=\frac{I^{\uparrow}-I^{\downarrow}}{I^{\uparrow}+I^{\downarrow}}$, where $I^{\uparrow / \downarrow}$ is either the intensity integrated over the energy range of the full majority/minority peak $(\bullet)$ or 
(a)

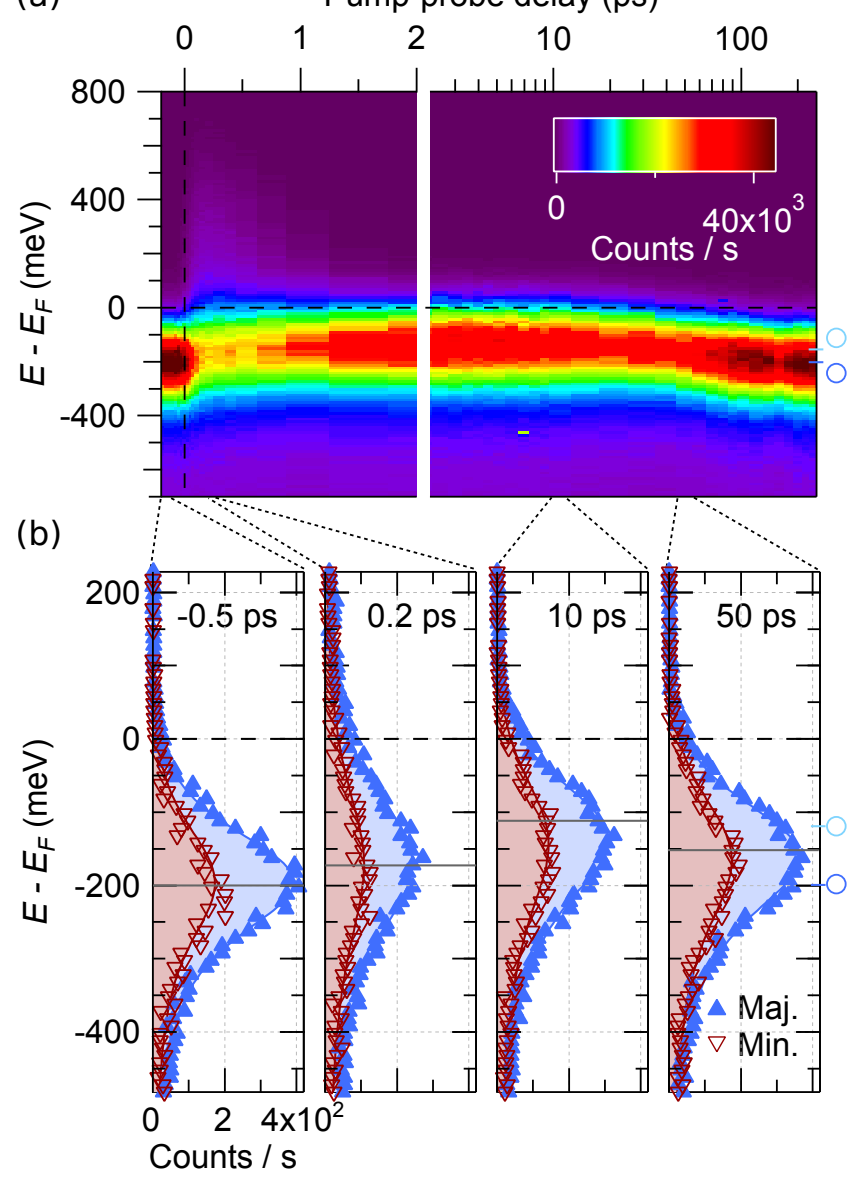

FIG. 2. (a) False color representation of spin-integrated photoemission spectra of the gadolinium $d_{z^{2}}^{\uparrow}$ surface state as a function of pump-probe delay. (b) Spin-resolved photoemission spectra at selected pump-probe delays. The spin polarization (asymmetry between majority $(\boldsymbol{\Delta})$ and minority $(\nabla)$ count rates) shows only weak changes in all four spectra.

the photoemission intensity measured at a single binding energy $(\circ, \circ)$. Integrating over the full peak yields more accurate values but also requires a lot more data acquisition-time and is thus more susceptible to changes of the surface quality. We therefore complemented the data by measurements at distinct energies with a significant spin polarization. Here we chose the maximal and minimal binding energy of the $d_{z^{2}}^{\uparrow}$ of $-200(\circ)$ and $-120 \mathrm{meV}(\circ)$ (indicated in Fig. 2 on the right ordinate) [42].

Figure 3 (a) shows the immediate increase of the electronic temperature up to $2,000 \mathrm{~K}$ within the temporal resolution of $70 \mathrm{fs}$. The subsequent temporal evolution of the temperature within the first picoseconds can be described (solid line) by an exponential decay that is overlayed by a rise in lattice temperature (dashed line). We thus assume that the electrons cool down predominantly transferring energy to the lattice at a time constant of $\tau_{\mathrm{E}}=0.6 \pm 0.1 \mathrm{ps}$. We find that the shift in binding en-

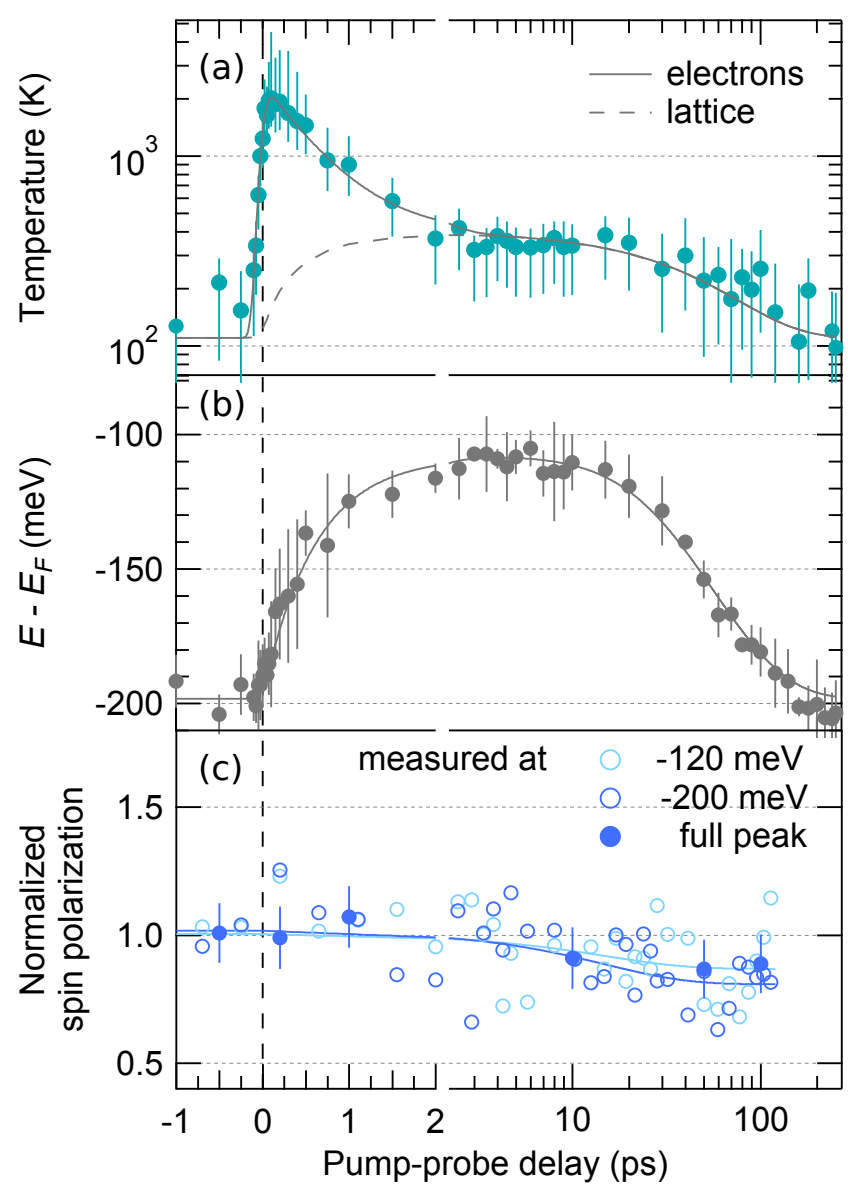

FIG. 3. Temporal evolution of (a) electronic temperature as obtained from the broadening of the Fermi edge, (b) binding energy, and (c) spin polarization of the $d_{z^{2}}^{\uparrow}$. The spin polarization integrated over the energy-range of the full peak $(\bullet)$ is complemented by data taken at $120 \mathrm{meV}$ ( $\circ$ ) and $200 \mathrm{meV}$ (॰) binding energy indicated in Fig. 2 on the right ordinate.

ergy (Fig. 3(b)) occurs with the same exponential time constant $\tau_{\mathrm{E}}$ and must therefore be induced by a heating of the lattice due to electron-phonon scattering. Thus, the peak position is determined by the lattice temperature. This is in perfect agreement with the results in the equilibrium phase transition ( $c f$. Fig.1). In contrast, the spin polarization itself shows in fact a much slower decrease of small magnitude. An exponential fit to the spin polarization results in a decay time of $\tau_{S}=15 \pm 8 \mathrm{ps}$ (dark and light blue lines for -200 and $-120 \mathrm{meV}$ ). This slow decrease can already be explained by spin-lattice coupling as has been suggested in Ref. 12 .

The evolutions of the spin polarization in the laserdriven experiment and in the thermal phase transition (filament heating) are compared in Fig. 4. The bottom axis shows the corresponding peak energy for each temperature in the equilibrium experiment $(\mathbf{-})$ and for each pump-probe delay in the laser-driven experiment $(\bullet, \circ)$ respectively. We find that upon optical excitation the 
peak position shifts to $E-E_{\mathrm{F}}=-110 \mathrm{meV}$ with almost no decrease in spin polarization. In the equilibrium phase transition, the $d_{z^{2}}^{\uparrow}$ already looses its spin polarization when it reaches the same energy, since this is when the sample is heated across $T_{\mathrm{C}}$ ( $c f$. Fig. 1 ). In the laserdriven experiment we observe a small reduction of the spin polarization to $\sim 0.9$, which is much slower than the changes in binding energy. The reduced spin polarization remains during the cooling of the lattice when the peak energy is already shifting back.

It has been reported for a photoemission experiment with a lower pump fluence of $1 \mathrm{~mJ} / \mathrm{cm}^{2}$ [19] that the $d_{z^{2}}^{\uparrow}$ shift has not much effect on the total exchange splitting of the surface state. Nevertheless, experiments applying fluences similar to that in our experiment, find a breakdown of magnetic signals like XMCD [14] or MOKE [15] occurring on the same timescale as the $d_{z^{2}}^{\uparrow}$ shift. Also the exchange splitting of the $(5 d 6 s)$ valence-bands decreases with the same time constant $[17,18]$. Therefore, we interpret the shift in our experiment as a change in exchange splitting, which is not accompanied by a reduction of spin polarization. We thus demonstrate that the response of the $\operatorname{Gd}(0001) d_{z^{2}}^{\uparrow}$ surface state to an ultrafast laser excitation is completely Stoner-like within the first picoseconds. While in the equilibrium phase transition Stoner and spin-mixing behavior occur simultaneously, in the laser-driven experiment the spin polarization decreases exponentially with $\tau_{\mathrm{S}}=15 \pm 8$ ps subsequent to the Stoner-like change of the binding energy with $\tau_{\mathrm{E}}=0.6 \pm 0.1 \mathrm{ps}$.

Three processes are reflected in the dynamics of the $d_{z^{2}}^{\uparrow}$ surface state. i) The fastest process, the direct heating of the electrons by the laser $(<70 \mathrm{fs})$, causes the fast response found in magnetic SHG [19, 21, 22]. According to Ref. 21 , the highly spin-polarized surface state contributes dominantly to the SHG process. Thus, its depopulation lowers the magnetic component of the signal. ii) Subsequently, energy is transferred from the electrons to the lattice thereby inducing the Stoner-like shift of $d_{z^{2}}^{\uparrow}$ with $\tau_{\mathrm{E}}=0.6 \pm 0.1 \mathrm{ps}$. MOKE depends on all possible transitions in the Brillouin zone [43]. It is therefore sensitive to the exchange splitting of the $(5 d 6 s)$ bands and hence shows a corresponding response time. It is however surprising that the XMCD contrast at the $M_{5}$ edge decreases on this timescale as well [14]. iii) XMCD and MOKE also reveal a second timescale in the ten picosecond regime similar to the response we find in the $d_{z^{2}}^{\uparrow}$ spin polarization. Recent results revealed that the MLD in photoemission from the $4 f$ core-levels decreases on the same slow ps-timescale [23]. This suggests that the spin polarization of the valence states is determined by the $4 f$ magnetic moment [44]. Such a demagnetization on two distinct timescales was already predicted by Koopmans et al. [45], but their model is solely based on a mean field description. It does not distinguish between exchange

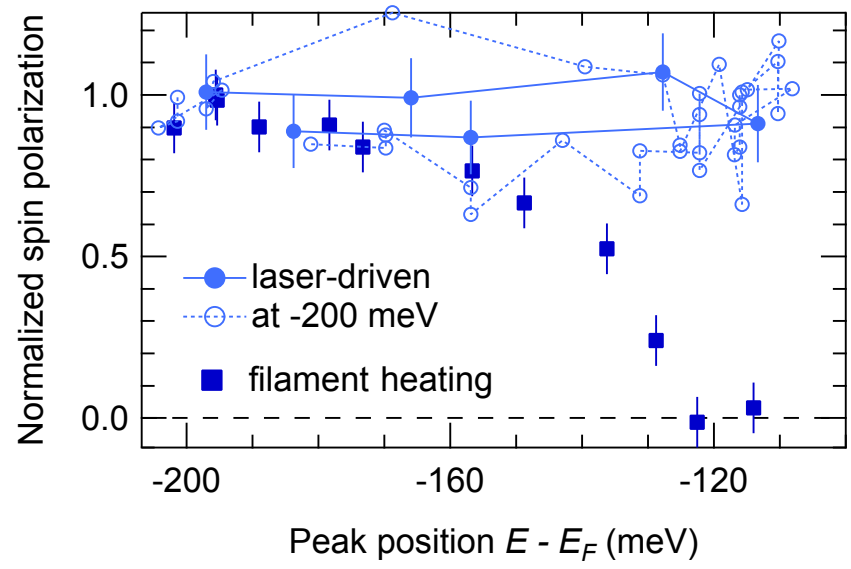

FIG. 4. Comparison of laser-driven $(\bullet, \circ)$ and thermal phase transition ( $\mathbf{\square})$. The spin polarization is plotted $v s$. the binding energy corresponding to a pump-probe delay or temperature, respectively. Open symbols ( $\circ$ ) display the spin polarization obtained at a single energy $(-200 \mathrm{meV})$. Filled symbols $(\bullet)$ show energy-integrated values for the full $d_{z^{2}}^{\uparrow}$ peak. The uncertainties of the peak position are shown in Figs. 1 and 3.

splitting and spin polarization, and hence misses important ingredients to describe the magnetization dynamics of $4 f$ metals.

It is reasonable to assume that the observed behavior is characteristic for $\mathrm{Gd}$, since spin-resolved photoemission measurements on $\mathrm{Fe}$ and Ni find an ultrafast breakdown of the overall spin polarization, though without orbital resolution [43, 46, 47].

In conclusion, we find that the response times of spin polarization and exchange splitting differ by more than one order of magnitude. This leads to a Stoner-like behavior of the surface state during the first picoseconds. We expect the huge difference between the Stoner and spin-mixing timescales in $\mathrm{Gd}$ to arise from the indirect exchange interaction. While the $(5 d 6 s)$ valence electrons are directly affected by the laser excitation leading to the decreasing exchange splitting, the $4 f$ spin system remains cold for a long time stabilizing the spin polarization [23]. In contrast, in the equilibrium phase transition, these processes can not be separated. Using ultrafast laser excitation, we are able to disentangle exchange splitting and spin polarization, which opens a completely new view on the magnetic phase transition.

We thank the Helmholtz Virtual Institute Dynamic Pathways in Multidimensional Landscapes and the DFG for financial support.

* Corresponding author: andres@physik.fu-berlin.de

[1] A. Cavalleri, C. Tóth, C. W. Siders, J. A. Squier, F. Ráksi, P. Forget, and J. C. Kieffer, Phys. Rev. Lett. 87, 237401 (2001). 
[2] L. Perfetti, P. A. Loukakos, M. Lisowski, U. Bovensiepen, H. Berger, S. Biermann, P. S. Cornaglia, A. Georges, and M. Wolf, Phys. Rev. Lett. 97, 067402 (2006).

[3] D. Fausti, R. I. Tobey, N. Dean, S. Kaiser, A. Dienst, M. C. Hoffmann, S. Pyon, T. Takayama, H. Takagi, and A. Cavalleri, Science 331 (2011), 10.1126/science.1197294.

[4] L. Stojchevska, I. Vaskivskyi, T. Mertelj, P. Kusar, D. Svetin, S. Brazovskii, and D. Mihailovic, Science 344 (2014), 10.1126/science.1241591.

[5] D. Wegkamp, M. Herzog, L. Xian, M. Gatti, P. Cudazzo, C. L. McGahan, R. E. Marvel, R. F. Haglund, A. Rubio, M. Wolf, and J. Stähler, Phys. Rev. Lett. 113, 216401 (2014).

[6] M. Dell'Angela, T. Anniyev, M. Beye, R. Coffee, A. Föhlisch, J. Gladh, T. Katayama, S. Kaya, O. Krupin, J. LaRue, A. Møgelhøj, D. Nordlund, J. K. Nørskov, H. Öberg, H. Ogasawara, H. Öström, L. G. M. Pettersson, W. F. Schlotter, J. A. Sellberg, F. Sorgenfrei, J. J. Turner, M. Wolf, W. Wurth, and A. Nilsson, Science 339, 1302 (2013).

[7] I. Radu, K. Vahaplar, C. Stamm, T. Kachel, N. Pontius, H. A. Dürr, T. A. Ostler, J. Barker, R. F. L. Evans, R. W. Chantrell, A. Tsukamoto, A. Itoh, A. Kirilyuk, T. Rasing, and A. V. Kimel, Nature 472, 205 (2011).

[8] C. D. Stanciu, F. Hansteen, A. V. Kimel, A. Kirilyuk, A. Tsukamoto, A. Itoh, and T. Rasing, Phys. Rev. Lett. 99, 047601 (2007)

[9] A. Kirilyuk, A. V. Kimel, and T. Rasing, Rev. Mod. Phys. 82, 2731 (2010).

[10] S. Mangin, M. Gottwald, C.-H. Lambert, D. Steil, V. Uhlír, L. Pang, M. Hehn, S. Alebrand, M. Cinchetti, G. Malinowski, Y. Fainman, M. Aeschlimann, , and E. E. Fullerton, Nature Mater. 13, 286 (2014)

[11] E. Beaurepaire, J.-C. Merle, A. Daunois, and J.-Y. Bigot, Phys. Rev. Lett. 76, 4250 (1996).

[12] A. Vaterlaus, T. Beutler, and F. Meier, Phys. Rev. Lett. 67, 3314 (1991), gd004.

[13] A. Melnikov, H. Prima-Garcia, M. Lisowski, T. Giessel, R. Weber, R. Schmidt, C. Gahl, N. M. Bulgakova, U. Bovensiepen, and M. Weinelt, Phys. Rev. Lett. 100 107202 (2008).

[14] M. Wietstruk, A. Melnikov, C. Stamm, T. Kachel, N. Pontius, M. Sultan, C. Gahl, M. Weinelt, H. A. Dürr, and U. Bovensiepen, Phys. Rev. Lett. 106, 127401 (2011).

[15] M. Sultan, U. Atxitia, A. Melnikov, O. ChubykaloFesenko, and U. Bovensiepen, Phys. Rev. B 85, 184407 (2012).

[16] M. Sultan, A. Melnikov, and U. Bovensiepen, Phys. Status Solidi B 248, 2323 (2011)

[17] R. Carley, K. Döbrich, B. Frietsch, C. Gahl, M. Teichmann, O. Schwarzkopf, P. Wernet, and M. Weinelt, Phys. Rev. Lett. 109, 057401 (2012).

[18] M. Teichmann, B. Frietsch, K. Döbrich, R. Carley, and M. Weinelt, Phys. Rev. B 91, 014425 (2015).

[19] M. Lisowski, P. A. Loukakos, A. Melnikov, I. Radu, L. Ungureanu, M. Wolf, and U. Bovensiepen, Phys. Rev. Lett. 95, 137402 (2005).

[20] P. A. Loukakos, M. Lisowski, G. Bihlmayer, S. Blügel, M. Wolf, and U. Bovensiepen, Phys. Rev. Lett. 98, 097401 (2007).

[21] A. Melnikov, I. Radu, U. Bovensiepen, O. Krupin, K. Starke, E. Matthias, and M. Wolf, Phys. Rev. Lett.
91, $227403(2003)$

[22] A. Melnikov, I. Radu, A. Povolotskiy, T. Wehling, A. Lichtenstein, and U. Bovensiepen, Journal Of Physics D-Applied Physics 41, 164004 (2008).

[23] B. Frietsch, J. Bowlan, R. Carley, M. Teichmann, S. Wienholdt, D. Hinzke, U. Nowak, K. Carva, P. M. Oppeneer, and M. Weinelt, Nat Commun 6, (2015).

[24] A. Winkelmann, D. Hartung, H. Engelhard, C.-T. Chiang, and J. Kirschner, Rev. Sci. Instrum. 79, 083303 (2008).

[25] B. Andres, P. Weiss, M. Wietstruk, and M. Weinelt, Journal of Physics: Condensed Matter 27, 015503 (2015).

[26] E. C. Stoner, Proceedings of the Royal Society of London. Series A, Mathematical and Physical Sciences 154, pp. 656 (1936).

[27] J. C. Slater, Phys. Rev. 49, 537 (1936).

[28] J. C. Slater, Phys. Rev. 49, 931 (1936).

[29] E. C. Stoner, Proceedings of the Royal Society of London. Series A, Mathematical and Physical Sciences 165, pp. 372 (1938).

[30] V. Korenman, J. L. Murray, and R. E. Prange, Phys. Rev. B 16, 4032 (1977).

[31] H. Capellmann, Zeitschrift für Physik B Condensed Matter 34, 29 (1979).

[32] H. Hasegawa, Journal of the Physical Society of Japan 46, 1504 (1979)

[33] R. E. Prange and V. Korenman, Phys. Rev. B 19, 4691 (1979).

[34] A. J. Pindor, J. Staunton, G. M. Stocks, and H. Winter, Journal of Physics F: Metal Physics 13, 979 (1983).

[35] M. Bode, M. Getzlaff, S. Heinze, R. Pascal, and R. Wiesendanger, Applied Physics A: Materials Science \& Processing 66, S121 (1998-03-01).

[36] M. Getzlaff, M. Bode, S. Heinze, R. Pascal, and R. Wiesendanger, Journal of Magnetism and Magnetic Materials 184, 155 (1998).

[37] M. Donath, B. Gubanka, and F. Passek, Phys. Rev. Lett. 77, 5138 (1996)

[38] E. Weschke, C. Schüssler-Langeheine, R. Meier, A. V. Fedorov, K. Starke, F. Hübinger, and G. Kaindl, Phys. Rev. Lett. 77, 3415 (1996).

[39] K. Maiti, M. C. Malagoli, A. Dallmeyer, and C. Carbone, Phys. Rev. Lett. 88, 167205 (2002).

[40] U. Bovensiepen, J. Phys.: Cond. Matter 19, 083201 (2007).

[41] See Supplemental Material at [URL will be inserted by publisher] for a decomposition of the fit.

[42] See Supplemental Material at [URL will be inserted by publisher] for the corresponding spin-resolved photoemission intensities.

[43] A. Weber, F. Pressacco, S. Günther, E. Mancini, P. M. Oppeneer, and C. H. Back, Phys. Rev. B 84, 132412 (2011)

[44] L. M. Sandratskii, Phys. Rev. B 90, 184406 (2014).

[45] B. Koopmans, G. Malinowski, F. Dalla Longa, D. Steiauf, M. Fahnle, T. Roth, M. Cinchetti, and M. Aeschlimann, Nat Mater 9, 259 (2010).

[46] A. Fognini, T. U. Michlmayr, G. Salvatella, C. Wetli, U. Ramsperger, T. Bähler, F. Sorgenfrei, M. Beye, A. Eschenlohr, N. Pontius, C. Stamm, F. Hieke, M. Dell'Angela, S. d. Jong, R. Kukreja, N. Gerasimova, V. Rybnikov, A. Al-Shemmary, H. Redlin, J. Raabe, A. Föhlisch, H. A. Dürr, W. Wurth, D. Pescia, A. Vaterlaus, and Y. Acremann, Applied Physics Letters 104, 
032402 (2014).

[47] A. Scholl, L. Baumgarten, R. Jacquemin, and W. Eberhardt, Phys. Rev. Lett. 79, 5146 (1997). 